\title{
GSM TDMA Frame Rate Internal Active Noise Cancellation
}

\author{
Ingvar Claesson \\ Blekinge Institute of Technology, SE 37225 Ronneby, Sweden
}

\author{
Andreas Nilsson \\ Ericsson Mobile Platform, SE 22100 Lund, Sweden
}

(Received 2 April 2002; accepted 11 August 2003)

\begin{abstract}
A common problem in the world's most widely-used cellular telephone system, the GSM system, is the interfering signal generated by the switching nature of TDMA cellular telephony in handheld and other terminals. Signals are sent as chunks of data, speech frames, equivalent to 160 samples of data corresponding to $20 \mathrm{~ms}$ at sampling rate of $8 \mathrm{kHz}$. This paper describes a study of two different software solutions designed to suppress such interference internally in the mobile handset. The methods are 1) notch filtering, which is multiplicative in frequency, and 2) subtractive noise cancellation, which is an alternative method employing correlators. The latter solution is a straigtforward, although somewhat unorthodox, application of "in-wire" active noise control. Since subtraction is performed directly in the time domain, and we have access to the state of the mobile, it is also possible to consider a recurring pause in the interference caused by the idle frame in the transmission, when the mobile listens to other base stations communicating. More complex control algorithms, based on the state of the communication between the handset and the base station, can be utilised.
\end{abstract}

\section{INTRODUCTION}

In GSM mobile telephony, it is a common problem that an interfering signal is introduced into the microphone signal when the mobile telephone is transmitting. This interfering signal is transmitted along with the speech signal to the receiver. Due to the humming sound of the interfering signal it is commonly referred to as the 'bumblebee.'

Since interleaving of data are utilised and since control data transmission is also necessary, the connection between transmitter/receiver frames and speech frames is somewhat complicated. Data from a speech frame of $20 \mathrm{~ms}$ are sent in several bursts, each occupying $1 / 8$ of a transmitting frame. The radio circuits are switched on and off with the radio access rate frequency. An electromagnetic field pulsating with this frequency and its harmonics disturbs its own microphone signal, as well as electronic equipment in the vicinity, producing in some cases an annoying periodic humming noise in the uplink speech from the handset to the base station.

The bumblebee is generated by the switching nature of TDMA cellular telephony, where the radio circuits are switched on and off. During the time the radio is switched on, known as the 'time slot,' the mobile telephone transmits its information by sending electromagnetic impulses. These impulses are induced in the microphone path and generate interference, which consists of the fundamental frequency and its harmonics. The fundamental switching rate is approximately $217 \mathrm{~Hz}$, or more specifically, 5200/(3 - 8) Hz, according to the GSM standard. ${ }^{1}$

Since the frequency components of the disturbing periodic humming noise are crystal-generated and accurately known, it is possible to estimate the cosine and the sine parts of these with correlators. This is easily done by correlating the microphone signal with sinusoids having the same crys- tal-generated frequencies as the disturbing frequencies. By generating the cosine and sine signals with correct signed amplitudes and then subtracting these from the microphone signal, the humming bumblebee is almost perfectly suppressed in the microphone signal. This is a classical example where in-wire subtractive active noise control is beneficial. ${ }^{2,3}$

Depending on the power level the mobile telephone is transmitting, how it is held and if one uses portable hands-free equipment or not, the amplitudes and phases of the fundamental and its harmonics will vary. When the mobile changes time slot, i.e., during a hand-over between base stations, the amplitudes and phases will also change abruptly.

Earlier solutions of this problem have utilised different hardware constructions, i.e., better placement of the components, usage of special electronics and microphones, reconstruction of analog parts, etc. However, this is expensive, time consuming and becomes increasingly difficult since the mobile telephones are constantly shrinking in size, thus causing the microphone to be situated closer to the transmitting antenna.

The solution to the problem presented in this paper makes use of the fact that the disturbance, after a Fourier series expansion, can be accurately described by a sum of sinusoids with well-defined frequencies. Two time domain software solutions are evaluated in this study to attenuate these frequency components of the digitised microphone signal directly in the base band by using synchronised correlators and notch filtering.

The best results were achieved by estimating the amount of the different sinusoids with correlators, and then subtracting these sinusoidal estimates from the microphone signal, as opposed to conventional notch filtering. This is an illustrative example of an application in which subtraction of disturbances, typical for active noise control, ${ }^{2,4}$ is suitable. 